\title{
Factors influencing quality online learning experiences
}

\author{
Ron Oliver and Jan Herrington \\ Edith Cowan University
}

\begin{abstract}
The gradual uptake of online learning as a delivery medium for higher education necessitates a shift in the metrics used to determine quality and success. While the attributes that have typically been used as benchmarks for quality in learning remain the same, the factors that impact on these factors have changed and it is these factors that form the focus of this paper. The aim of this paper is to explore from both a conceptual and grounded perspective, the defining factors in the quality of students' learning experiences and to consider how can these be optimised in online learning settings.
\end{abstract}

Key words: online learning, quality

\section{DETERMINING QUALITY IN LEARNING}

If we consider a student's learning experience as a composite of all experiences associated with completion of a formal course of study, there are a number of discrete and connected elements that can be addressed which will have impacted on the notion of quality in relation to the learning process. In assessing the overall quality of the learning experience, it is necessary to consider the quality of each of these elements as limitations in any will necessarily impact on the student's learning experiences and subsequent learning outcomes (eg. Bonk \& Dennen, 2001).

The factors that one would typically consider in such explorations would include such items as: the administration of the course, the nature of the course itself, its objectives and aims and how well these fit within the overall program in which it sits, the context in which the learning is undertaken, the level of engagement associated with learning in the course, the scope and

The original version of this chapter was revised: The copyright line was incorrect. This has been corrected. The Erratum to this chapter is available at DOI: 10.1007/978-0-387-35700-3_33 
extent of the resources and the forms of learning support. Often these factors are assessed through questionnaires canvassing student perceptions and impressions (eg. Devlin, 2002)

When looking from first principles, a quality learning experience supports the forms of conceptual change that leaves the learner with some planned and intended form of capability. A quality learning experience needs to arrive at this endpoint in an efficient manner. It needs to provide the learner with appropriate forms of encouragement, cognitive engagement and feedback that lead from an initial to an intended state underpinned by a knowledge base that provides context and relevance (eg. Grabinger, 1996).

\subsection{Quality in learning}

A number of writers have sought to describe quality learning experiences from a cognitive perspective. For example, Savery \& Duffy (1995) argue that there are four principles that necessarily underpin effective learning and derive these principles from learning theories that support knowledge construction as the descriptors of how students learn. Savery and Duffy argue that quality learning occurs when: learning is an active and engaged process; learning is a process of constructing knowledge; learners function at a metacognitive level; and learning involves social negotiation.

Others have sought to describe quality learning experiences from a learner's perspective. Boud \& Prosser (in press) argue that quality learning is achieved when learners are involved in activities which: engage learners by supporting their intent and expectations; challenge learners to question assumptions, to discern variations, to go beyond what is provided and take ownership of the learning process; acknowledge context through the provision of appropriate problems and the situating of learning in meaningful contexts; and involve practice that enable learners to demonstrate what has been learned, to gain feedback as they progress and reflect on the experience and develop confidence.

\subsection{Quality in teaching}

A number of writers have described quality learning experiences from the teacher's perspective, and use the notion of learning support as an important determinant of quality learning. McLoughlin (2002), for example, argues the need for appropriate scaffolding for the distant learner and suggests a number of dimensions that can be applied in determining the capability of scaffolds to effectively support the distant learner. The dimensions include 
such attributes as the need for scaffolds to be oriented towards the attainment of students' goals, the adaptability of scaffolds to cater in different ways for different learners, the need for scaffolds to be accessible at the point of need, the need for scaffolds to be aligned with intended outcomes and to provide experiential value rather than exposure and access to inert knowledge.

\subsection{Quality in courseware and delivery}

Alternative views describe quality learning from a designer's perspective. Oliver (1999) suggests a quality learning environment is characterised by purposeful and informed design of appropriate learning tasks as the context for learning, learning resources that provide the underpinning knowledge and learning supports that scaffold and support learning. Herrington et al. (2001) provide a checklist based on these principles that use determinations of pedagogy, resources and delivery strategies as the means for assessing the potential and quality of any online learning setting. In a similar fashion, Sims, Dobbs and Hand (2002) argue that quality learning experiences in technology-mediated distance settings such as online learning are determined by issues of quality in the design and delivery of the online setting.

\section{MEASURING QUALITY IN TEACHING AND LEARNING}

The existence of so many possible ways to explore and evaluate student learning experiences in online settings creates a number of difficulties for institutions looking to provide some form of quality assurance for their online activities. The solution that many institutions are adopting to this complex issue, is to use student feedback as a prime quality indicator and more and more today, we are seeing student perceptions being used as the principal data source for quality assurance processes associated with teaching. The use of student perceptions as a feedback source presents a number of opportunities. While student perceptions may not provide empirical evidence of learning outcomes, they can provide evidence of the amenities and impediments of the learning setting and as such are a useful resource for this purpose. In the local context, with Government using student perceptions in the form of the Course Experience Questionnaire as a measure and yardstick of program success, such an approach is well grounded in practice and policy for institutions to follow. 


\section{FACTORS INFLUENCING QUALITY ONLINE LEARNING EXPERIENCES}

At Edith Cowan University we have been delivering courses online for many years and have been exploring student experiences and learning outcomes from the online environments across that period in a variety of forms. One course of note, the Graduate Certificate in Online Learning (GCOLL), has been running for several years now and most units have had a number of repetitions. As part of our evaluation process we have been monitoring student feedback (perceptions) and using a process of continuous improvement to revise and update the various units. Through our inquiries and investigations we have identified a number of items which appear to have had a significant impact on students' learning experiences. We have based much of our redevelopment work with the units in seeking strategies for maximising the amenities and minimising the problems to provide students with quality learning experience.

\subsection{Implementation strategies}

The area that appears from our experiences to contribute the most to students' dissatisfaction and perceptions of a diminished learning environment is the delivery of the course to the remote learner. In the main this area tends to be associated with the technology supporting the learning environment and students' experiences with it. An independent online learner requires a relatively high level of technical skills to ensure a smooth and problem-free learning experience. It is almost impossible to anticipate the technical problems that can confront learners. But it is not difficult to imagine the impact of technical problems on students' learning experiences.

\subsubsection{Providing technical support}

In anticipation of potential technical problems associated with delivering units online, with the planning of the GCOLL we established a number of procedures and protocols that were intended to minimise downtime and to assist students for whom learning with technology might be a new experience. We have always been relatively comfortable with our planning and the contingencies that we have in place. Despite our best efforts, we continually experience technical problems that cause a number of the students serious headaches and which lessen the quality of their learning experiences especially in the first few weeks of the course. 


\subsubsection{Administrative support}

In campus-based face to face teaching, students tend to recognise the roles of the various agencies within the institution responsible for the delivery of services that support their learning. A number of authors have cautioned institutions to ensure that they are aware of the provision of services to their online students at similar levels to students who study on campus. Typically servicing is never as good and many students express dissatisfaction with their learning experiences based on shortcomings in this area.

From a quality perspective, getting the delivery right and creating an online learning experience for students which is devoid of any technical problems or concerns, should be the goal of all online delivery. Given the myriad of possible ways for delivery to falter, this would appear to be an area that will always need constant attention and proactive problem solving in the quest for excellence.

\subsection{Learning skills}

Our delivery of the GCOLL has demonstrated the need for a number of learning skills among online learners, skills which frequently are limited when the students commence their online studies. One skill which learners in online settings frequently need to employ is that of being able to work collaboratively in groups and teams. In the GCOLL, the use of the group is intended to reduce the work needed by students over an independent completion. At the same time, learning in a group provides opportunities for communication, discourse and dialogue likely to strengthen learning and understanding. When faced with group work, many students will work as a crowd rather than as a crew. A number of students report a large amount of their effort being directed towards managing the group rather than being directed towards their learning and learning outcomes. In such instances, students will often see the setting as an impediment to their learning rather than as an aid or a support and will sense dissatisfaction with the experience and its outcomes.

\subsubsection{Inquiry learning}

Our experience in the GCOLL has suggested that novice online learners are often very uncertain and very discomforted with undertaking process to seek solutions to open-ended tasks. Students have come to expect from their 
teachers, assistance of any form and of any scope when requested. In the case of problem solving, we have seen many students finding difficulties in implementing processes.

\subsubsection{Information overload}

When faced with a task and a set information space, we have found most students are quite comfortable in the approaches they take. When the information space is opened up, however, and there is more information than required, some students will fall into traps. They do not know when to stop their information seeking and feel compelled to use the entire information source.

Helping learners to become good online learners demands some proactive measures within and beyond the discrete courses which learners undertake. We are now seeing a number of supports being developed by institutions and organizations to assist their students to develop skills in online learning. Learning in online settings can require skills from the learner that might not ordinarily be present. Learners accustomed to face to face settings frequently find themselves in learning environments that have high degrees of structure and form. The forms of activities provided by the teachers are directed and provide little scope for personal decision making. Online learning on the other hand, provides teachers with opportunities for many different forms of learner activity and students are often expected to be able to accommodate the requirements of alternative learning designs with little training or reskilling.

\subsection{Teaching Skills}

In early days, institutions were of the belief that online courses were going to provide huge economies for course delivery and that online learning would see large reductions in the need for teacher involvement in course delivery. Practice has demonstrated almost an opposite outcome. Online teachers are seeing their role in the learning environment requiring high levels of involvement and with that involvement, they are seeing the need for a set of skills and knowledge not likely to have developed from conventional teaching forms. Students' learning experiences appear in contemporary settings to be influenced to a large degree by the activities and interventions of the online teacher, and feedback from our courses has indicated a number of ways in which particular skills and expertise on the part of the teacher can help to ensure a quality learning experience. 


\subsubsection{Supporting community development}

Online learning is enhanced considerably when the learners are able to establish some from of community through the learning process. The opportunities from the community derive from the learning achieved through the process of articulation, discussion and information sharing. It takes a degree of skill on the part of the online teacher to be able to create a community within an online setting.

\subsubsection{Managing expectations}

Online students tend to spend strange hours on their computers and appreciate that their teachers are frequently online as well. Students will be quite happy to email their teachers at any stage to seek help or guidance and most teachers would encourage this. Students, on the other hand, expect reasonably timely replies. This can become a trap for the online teacher who may not have the opportunity for frequent communication. Likewise, students will contribute discussion points and comments to the public fora and expect to see some responses in a timely fashion.

\subsubsection{Task selection}

The design of many parts of conventional learning settings have not been completed with a view to learning outcomes but more with a view to current and perceived best practice. For example, the provision of pages of text as replications of lectures is often devoid of any deliberate learning design yet the pages are used as organising elements for course presentation. The use of discussion boards as areas for public comment are often linked to mandatory forms and amounts of contribution. This form of activity is difficult to motivate if it is not seen to contribute to the aims and intentions of the learning.

\section{SUMMARY AND CONCLUSIONS}

If we believe most descriptions of evaluation strategies of online learning environments, the most influential factors in determining the quality of students' learning experiences would appear to be the resources and materials that have been prepared for the online course (e.g. Neumann, 1998). While this aspect of an online course is very important and a necessary component of a successful course, our experiences have tended to reveal that other factors of often greater importance are those associated with 
the delivery and implementation of the learning setting. The implementation processes and the quality of the facilitation provided by the online teacher play significant roles in students' perceptions of the quality of their learning experience. These factors are often independent of the course themselves and frequently are items for which least planning and development are provided. Institutions looking to develop their expertise and capability in online and elearning would do well to ensure that as well as planning and designing quality materials for their online courses, they also plan to ensure quality is achieved in the other equally, if not more important, aspects of the delivery process relating to administration and facilitation of the learning setting.

\section{REFERENCES}

ANTA (2002). http://www.tafe.net/learnscope/rsg

Bonk, C. and V. Dennen (2001). Framework for frameworks in Web instruction: Fostering research, design, benchmarks, training and pedagogy. In M. Moore. and B. Anderson (Eds) Handbook of American Distance Education.. Mahwah:NJ, Lawrence Erlbaum.

Boud, D. \& Prosser, M. (in press). Appraising new technologies for learning: a framework for evaluation and redevelopment. Educational Media International

McLoughlin, C. (2002). Learner support in distance and networked learning environments: Ten dimensions for successful design. Distance Education 23(2): 149-162.

Devlin, M. (2002). An improved questionnaire for gathering student perceptions of teaching and learning. Higher Education Research and Development 21(2): 289-304.

Grabinger, S. (1996). Rich environments for active learning. In D. Jonassen (Ed.) Handbook of Research for Educational Communications and Technology. New York: MacMillan Library Reference, 665-692.

Herrington, A., Oliver, R., Herrington, J., Stoney, S. \& Willis, J. (2001). Quality assurance of online courses: The development of an instrument to audit online units. In G.Kennedy, M.

Keppell, C. McNaught \& T. Petrovic (Eds), Meeting at the Crossroads, Proceedings of ASCILTE, 2001, 263-270. Melbourne, The University of Melbourne.

Mioduser, D., R. Nachmias, et al. (1999). Web-based learning environments: Current states and emerging trends. Ed-Media 1999. World Conference on Educational Multimedia, Hypermedia and Telecommunications, Seattle, USA, Association for the Advancement of Computers in Education.

Neumann, P. (1998). Risks of e-education. Communications of the ACM 40(10): 136.

Oliver, R. (1999). Exploring strategies for on-line teaching and learning. Distance Education, 20(2): 240-254.

Savery, J. \& Duffy, T. (1995). Problem-based learning: An instructional model and its constructivist framework. Educational Technology, 35(5): 31-38.

Sims, R., Dobbs, G, \& Hand, T. Enhancing quality in online learning: Scaffolding planning and design through proactive evaluation. Distance Education, 23(2): 2002. 